\title{
Cooperation Protocol for the Uplink of Relay Assisted Cellular Networks
}

\author{
Mihai P. Stef, Zsolt A. Polgar and Vasile Bota \\ Telecommunications Department \\ Technical University of Cluj Napoca \\ Cluj Napoca, Romania \\ E-mail: Mihai.Stef@com.utcluj.ro
}

\begin{abstract}
The paper proposes a network coded cooperation protocol for the uplink transmission of relay assisted cellular networks characterized by non-ideal source - relay links. The proposed protocol employs Reed Solomon codes as network codes in order to improve the Block Error Rate (BLER) and Bit Error Rate (BER) performance over the slow varying fading channels. The paper presents also an algorithm for the computation of the BLER ensured by the cooperation protocol when the cooperative links are modeled as Block Erasure Channels (BLEC), as well as an analysis of the performance obtained, showing the significant improvement brought by the employment of RS network codes.
\end{abstract}

Keywords-cooperative transmission, Reed Solomon codes, block fading, network coding

\section{INTRODUCTION}

Relaying and cooperation between users terminals are considered as some of the most promising approaches for improving the performance of wireless communications. Future standards like LTE Advanced and $802.16 \mathrm{~m}$ include also relay based cooperative communications in order to increase the throughput or to extend the coverage area of the cell. Most of the current studies concentrate on scenarios in which one or several relay nodes $(\mathrm{RN})$ serve one user terminal (UT) [1], [2], [3]. Different approaches based on the use of network coding (NC) techniques combined with channel coding can be found in literature, e.g. [4], [5], but these proposals are also limited to scenarios in which one relay node helps two user terminals.

The employment of NC-based cooperation techniques in wireless networks opens the door for more complex cooperative scenarios, like Multiple Source Multiple Relay (MSMR) cluster-based cooperation. This approach received a significant attention [6], [7], [8] several cooperation scenarios being defined in recent literature. This approach is expected to bring significant performance improvements, at the expense of increased system complexity

Another interesting solution for cluster-based cooperation is the employment of modern graph based codes, like LDPC, by matching the code graph on the network graph, solution proposed in [7] for Multiple Source Multiple Relay cooperation topologies. This approach has also several drawbacks. It is well known that the performance of the graph based codes, like LDPC, strongly depends on graph parameters, like cycles or stopping sets. For small graphs it is difficult to ensure good properties in terms of these parameters. This leads to the need of a large number of members which cooperate in order to ensure good graphs, significantly increasing the cooperation protocol complexity.

This paper proposes a NC-based cooperation protocol for the uplink transmission in clusters served only by one relay and with non-ideal source-relay links. The proposed protocol uses Reed Solomon (RS) codes [9] as network code. The protocol is mainly intended to improve the poor "waterfall" behavior [10] exhibited by channel codes, like turbo codes and LDPC codes, under slow fading channel conditions. This protocol could allow a simpler implementation and integration in current wireless systems in what concerns the required signaling and resource allocation. A method to compute the Block Error Rate (BLER) ensured by the proposed cooperation protocol is also provided.

The structure of the paper is the following: section 2 briefly describes the system model, section 3 presents the proposed cluster-based coded cooperation protocol employing ReedSolomon codes for the uplink transmission, section 4 proposes a method to compute the BLER ensured by the network code when the cooperative links are modeled as BLECs and also analyzes the provided BLER performance, while section 5 analyzes the performance of the cooperative uplink transmission, considering both the channel and the cluster code. Finally, section 6 concludes the paper.

\section{SYSTEM MODEL}

Figure 1. (left) presents a possible setup of a relay assisted cell with four fixed RNs. Each RN can serve more than one UT, and one UT can be served by more than one RN. Each RN together with the $N_{u}$ user terminals that it serves, $\mathrm{U}=\left\{\mathrm{UT}_{1}\right.$, $\left.\mathrm{UT}_{2}, \ldots, \mathrm{UT}_{\mathrm{Nu}}\right\}$, form a cooperation cluster. The topology under consideration is a centralized one, i.e. the base station (BS) knows all the cooperation clusters within the cell and each RN knows in which cooperation cluster it is located. Figure 1. (right) presents a possible cluster configuration for the uplink transmission, including four UTs.

All UTs direct channels (UTs-BS) and the relay channel (RN-BS) are affected by quasi-static Rayleigh fading and AWGN. For each link, UTs-BS and RNs-BS, the channel output for the $\mathrm{i}^{\text {th }}$ input sample is given by (1).

This work was partially funded by the European Union within FP7-ICT215477 CODIV Project. 


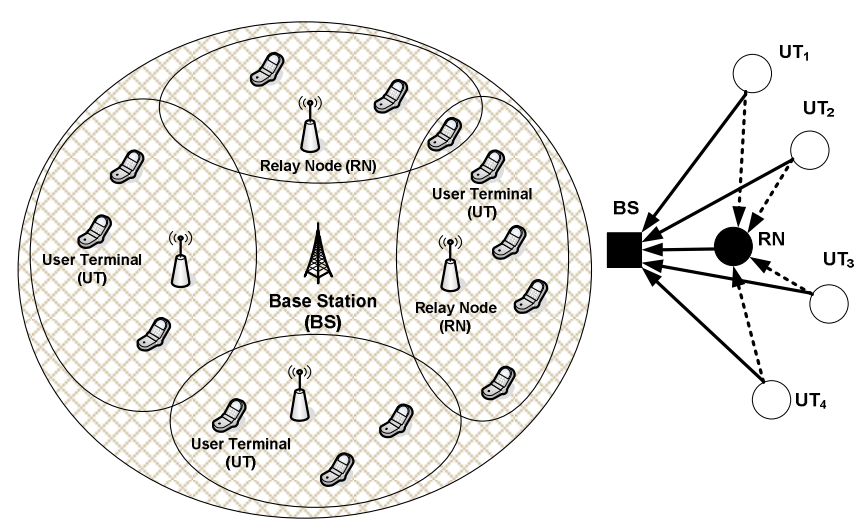

Figure 1. Relay assisted cellular network (left); Example of a cooperative cluster (right)

$$
y_{i}=h_{i} \cdot x_{i}+n_{i}
$$

where $x_{i} \in\{-1,1\}$ represents the channel input, using BPSK signaling; $h_{i}$ is the Rayleigh distributed channel coefficient, constant during a data block and constrained by the condition $E\left\{h_{i}^{2}\right\}=1$, while $n_{i}$ represents the normally distributed noise with zero mean and variance $\sigma=\sqrt{10^{S N R / 10}}$, where SNR represents the signal to noise ratio, expressed in $\mathrm{dB}$.

For the simplicity of the analysis each of the $\mathrm{UT}_{\mathrm{i}}-\mathrm{RN}$ links is modeled by the cooperation probability, $P_{\text {coop }}^{U T_{i}}$, representing the probability that the RN decodes correctly the $\mathrm{UT}_{\mathrm{i}}$ data block. $P_{\text {coop }}^{U T_{i}}$ depends on the block error rate (BLER) on the $\mathrm{UT}_{\mathrm{i}}$-RN link.

\section{REED SOLOMON CODE BASED COOPERATION PROTOCOL FOR THE UPLINK TRANSMISSION}

The proposed protocol for Multiple Source (MS) cooperation clusters is a two phase protocol operating as follows: in the first phase of cooperation each UT FECencodes its $N_{i}$ information bits using a rate $R_{i}$ code. The $N c_{i^{-}}$ long coded blocks obtained are broadcasted to the BS over the $\mathrm{UT}_{\mathrm{i}}-\mathrm{BS}$ links and to the cluster's $(\mathrm{RN})$ over the $\mathrm{UT}_{\mathrm{i}}-\mathrm{RN}$ links. In the second phase of cooperation the $\mathrm{RN}$ encodes the information blocks generated by the $\mathrm{UT}_{\mathrm{i}}-\mathrm{RN}$ links channel decoders using the network code. The additional, $N_{\text {check }}$, check blocks (generated by the network encoder) are employed to recover the erroneous data blocks received by the BS from the UTs over the direct links.

The protocol involves two separate level of coding, namely:

1. The first level of coding is represented by the channel code employed on each link of the cooperation cluster. This code will have to ensure quasi error-free UTs-RN links.

2. The second level of coding is represented by the employed network code, i.e. Reed-Solomon. The cluster code is applied only to the UTs' information bits.

The reason for employing RS codes as network codes is related to the good error and erasure correcting capabilities for small codeword lengths. The employed RS code has to be selected according to the number of UTs of the cooperation cluster and to the number of check blocks transmitted by the $\mathrm{RN}$. The parameters of the selected $\mathrm{RS}(\mathrm{n}, \mathrm{k})$ code have to satisfy the following inequalities:

$$
\left\{\begin{array}{l}
k \geq|U| \\
n-k \geq N_{\text {check }}
\end{array}\right.
$$

The length of the UTs' information blocks need to be equal at the input of the network encoder. If different information block lengths are employed by the UTs, the network encoder should be designed for the maximum length block and the shorter blocks will be padded with zeros. It is also necessary to ensure that the information bits applied to the network encoder can be grouped in an integer number of symbols, in order to employ the RS code. The number of zero padding bits for each block is computed according to:

$$
N z_{i}=\left[\max _{k}\left(N_{k}\right)-N_{i}\right]+\left[M-\max _{k}\left(N_{k}\right) \% M\right] \% M
$$

where $M$ represents the size of the RS symbols, $M=\log _{2}(n+1)$ and $\%$ represents the modulo operator.

After equalizing the length of the information blocks the RN performs the network encoding. The check blocks (symbols) of the network code are generated by encoding one symbol from all user data blocks at a time. The obtained check blocks are channel encoded and then are sent over the RN-BS channel. The network encoding process performed in the cooperation cluster is presented in Figure 2.

The BS separately channel decodes the blocks received from the UTs and from the RN and checks if the blocks are correct or not. If some of these blocks are erroneous then the network decoder will try to recover them.

The employed network code can correct errors or erasures, but only the erasure recovery property of the code is used in the proposed protocol. Figure 3 illustrates the processing performed by the base station.

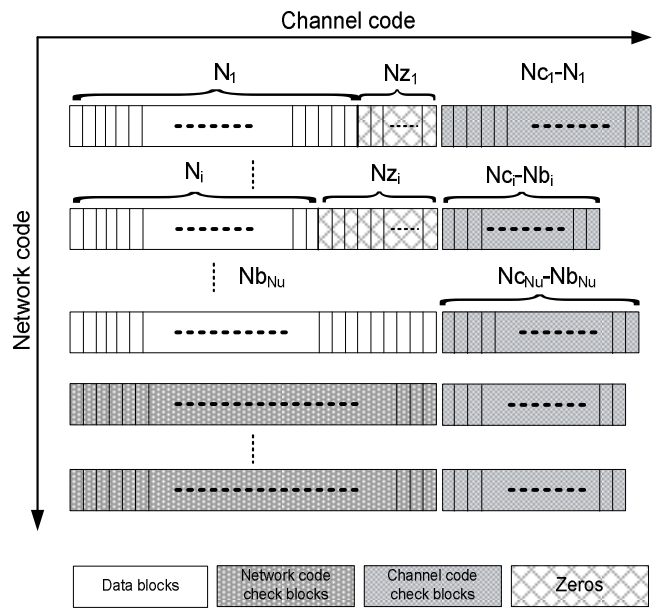

Figure 2. Cluster-based coded cooperation protocol for relay assisted cellular network 


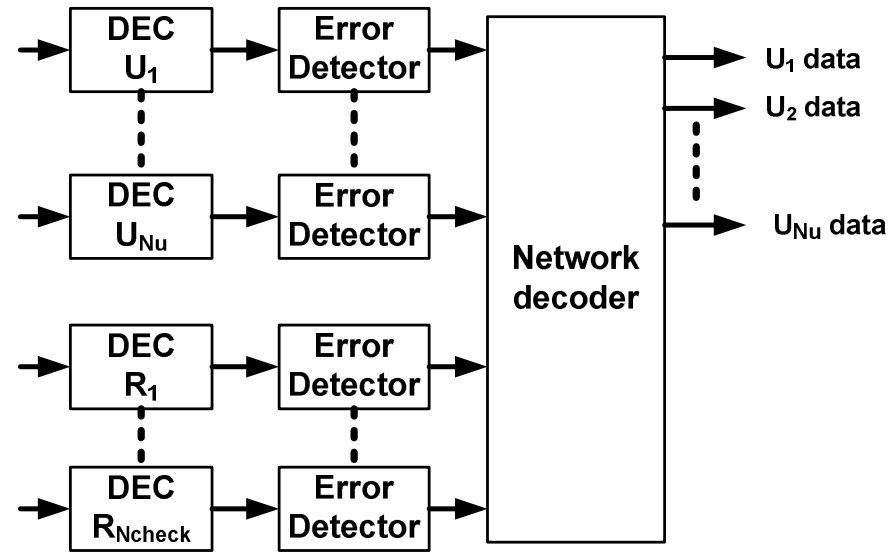

Figure 3. Block schematic of the decoding process performed by the base station

The protocol considers error affected UT - RN links, where the relay node eliminates the blocks received with errors. The BS has to know which blocks are involved in cooperation. As a result, it is necessary to update the cluster members list every cooperation period. To achieve this, each $\mathrm{RN}$ has to inform the $\mathrm{BS}$ which information blocks are used to compute the network code's check blocks. A one bit length field is sufficient for each user for this signaling operation.

\section{ANALYSIS OF THE RS BASED CLUSTER COOPERATION PROTOCOL}

In order to analyze the protocol, the $N_{u}+N_{\text {check }}$ blocks generated by the members of a cluster and processed at the BS are represented by the BL set as it follows:

$$
B L=\underbrace{\left\{b l_{k}(R N) \mid k=\overline{\left.1, N_{\text {check }}\right\}}\right.}_{B L R} \cup \underbrace{\left\{b l\left(U T_{i}\right) \mid U T_{i} \in U\right\}}_{B L U}
$$

where the $b l_{k}(R N)$ represent the $N_{\text {check }}$ blocks transmitted by the $\mathrm{RN}$, while the $b l\left(U T_{i}\right)$ represents the blocks transmitted by the cooperation cluster's UTs.

Because the check blocks computed by the RN are transmitted over the RN-BS they have the same error probability. The BLER of the link experienced by each block is denoted with $B L E R($ block $)=B L E R(b l(x))=B L E R_{x-B S}$.

\section{A. BLER computation of the RS-based protocol}

The BLER computation method of the proposed cluster cooperation protocol has to take into account the cooperation probabilities of the cluster's UTs, which are related to the BLERs on the UT-RN links, and the direct link's BLER of each UT.

Due to the fact that, in realistic conditions, the UTs have cooperation probabilities smaller than one, the number of UTs involved in the cooperation process varies over time, and a mean value of the number of cooperating UTs can be computed as:

$$
N U_{\text {mean }}=\sum_{i=1}^{N_{u}} p_{i} \cdot i
$$

where $p_{i}$ is the probability that a number of $i$ user terminal blocks are correctly received by the relay and are used to compute the check blocks. This probability depends on the cooperation probability of each user terminal.

$$
p_{i}=\sum_{U^{\prime} \in U^{(i)}}\left(\prod_{u t \in U^{\prime}} P_{c o o p}^{u t} \cdot \prod_{u t \in U^{\prime} \backslash U^{\prime}}\left(1-P_{c o o p}^{u t}\right)\right)
$$

where $U^{(i)}$ is the family of i-long subsets of the $U$ set, defined in section 2, and $P_{\text {coop }}^{u t}$ represents the probability that the user's data block is correctly received by its serving relay.

The probability that a certain data block cannot be recovered at the $\mathrm{BS}$ (the destination node) depends on the number of cooperating UTs at that moment. A solution is to compute this probability based on the mean number of cooperating UTs. Because this mean value is not always an integer number, an upper and a lower bounds should be used:

$$
N U_{\text {lower }}=\left\lfloor N U_{\text {mean }}\right\rfloor \quad N U_{\text {upper }}=\left\lceil N U_{\text {mean }}\right\rceil
$$

If the cooperation probability is lower than one, at least one of the $N U_{\text {lower }}$ and $N U_{\text {upper }}$ values will be different than the total number of the cluster's UTs. In the particular case when all UTs have identical cooperation probabilities and direct links' BLERs, the removed nodes can be selected randomly. The node selection problem in the case of nonidentical cooperation probabilities and direct link BLERs needs further investigations.

The $\mathrm{BLU}_{\text {lower }}$ and $B L U_{\text {upper }}$ subsets of the BLU set, having lengths $N U_{\text {lower }}$ and $N U_{\text {upper }}$, are selected randomly in the particular situation considered above, and we have:

$$
B L_{\text {lower }}=B L U_{\text {lower }} \cup B L R ; B L_{\text {upper }}=B L U_{\text {upper }} \cup B L R
$$

where the BLR and the BLU sets are defined in (4)

The lower and upper bounds of the non-recovery (nondecoding) probability, $\mathrm{PE}_{\mathrm{bl}(\mathrm{UTi})}$, are computed as in (11) where $\mathrm{BL}^{(\mathrm{k})}{ }_{\text {lower }}$ and $\mathrm{BL}^{(\mathrm{k})}$ upper represent the family of $\mathrm{k}$-long subsets of $\mathrm{BL}_{\text {lower }}$ respectively $\mathrm{BL}_{\text {upper }}$ sets. The non-recovery probability can be computed by linearly interpolating the lower and upper bounds given by (11), as follows:

$$
\begin{aligned}
P E_{b l\left(U T_{i}\right)} & =\left(N U_{\text {mean }}-N U_{\text {lower }}\right) \cdot P E_{b l\left(U T_{i}\right)}^{\text {lower }}+ \\
& +\left(N U_{\text {upper }}-N U_{\text {mean }}\right) \cdot P E_{b l\left(U T_{i}\right)}^{u p p e r}
\end{aligned}
$$

Finally the block error rate of a user terminal $U_{i}$ is obtained by taking into account the cooperation probability, the BLER on the direct link and the non-recovery probability:

$$
B L E R_{U T_{i}}=\left\{\begin{array}{l}
\left(1-P_{\text {coop }}^{U T_{i}}\right) \cdot B L E R_{U T_{i}-B S}+P_{\text {coop }}^{U T_{i}} \cdot P E_{b l\left(U T_{i}\right)}, P_{\text {coop }}^{U T_{i}} \neq 1 \\
P E_{b l\left(U T_{i}\right)}^{\text {lower }}=P E_{b l\left(U T_{i}\right)}^{u p p e r}, P_{\text {coop }}^{U T_{i}}=1
\end{array}\right.
$$




$$
\begin{gathered}
P E_{b l\left(U T_{i}\right)}^{\text {lower }}=\sum_{k=N_{\text {check }}^{R N}+1}^{N U_{l o w e r}+N_{\text {check }}^{R N}}\left(\sum_{\substack{B L^{\prime} \in B L_{\text {lower }}^{(k)} \\
b l\left(U T_{i}\right) \in B L^{\prime}}}\left(\prod_{b l \in B L^{\prime}} B L E R(b l) \cdot \prod_{b l \in B L_{\text {lower }} \backslash B L^{\prime}}(1-B L E R(b l))\right)\right) \\
P E_{b l\left(U T_{i}\right)}^{u \text { pper }}=\sum_{k=N_{\text {check }}^{R N}+1}^{N U_{\text {upper }}+N_{\text {check }}^{R N}}\left(\sum_{\substack{B L^{\prime} \in B L_{\text {upper }}^{(k)} \\
b l\left(U T_{i}\right) \in B L^{\prime}}}\left(\prod_{b l \in B L^{\prime}} B L E R(b l) \cdot \prod_{b l \in B L_{\text {upper }}\left(B L^{\prime}\right.}(1-B L E R(b l))\right)\right)
\end{gathered}
$$

The performance improvement is shown by the BLER gain parameter, $\mathrm{G}_{\mathrm{BLER}}$, defined as the ratio between the direct link's BLER and the BLER ensured by the cooperation protocol. The BLER gain $G_{B L E R}$ indicates how many times the direct transmission BLER is decreased by the cooperation scheme.

$$
\begin{aligned}
G_{B L E R}^{U T_{i}} & =\frac{B L E R_{U T_{i}-B S}}{B L E R_{U T_{i}}^{c o o p}} \\
& =\frac{B L E R_{U T_{i}-B S}}{\left(1-P_{\text {coop }}^{U T_{i}}\right) \cdot B L E R_{U T_{i}-B S}+P_{\text {coop }}^{U T_{i}} \cdot P E_{b l\left(U T_{i}\right)}}
\end{aligned}
$$

The maximum BLER gain is achieved by the cooperation scheme when the non-recovery probability equals zero. In this case the BLER gain depends only on the cooperation probability, as shown by the following equation:

$$
\operatorname{Gmax}_{B L E R}^{U T_{i}}=\frac{B L E R_{U T_{i}-B S}}{\left(1-P_{\text {coop }}^{U T_{i}}\right) \cdot B L E R_{U T_{i}-B S}}=\frac{1}{1-P_{\text {coop }}^{U T_{i}}}
$$

For cooperation probabilities that are different from one, the maximum BLER gain could be an important parameter of the channel and network code design algorithms.

\section{B. Analysis of the BLER performance of the RS-coded cooperation protocol}

Figure 4. presents the computed BLER performance of a cooperation cluster having the parameters given in Table I and different cooperation probabilities. The results are shown on a BLER vs. BLER plot, which has the BLER ensured by the channel code on the $\mathrm{X}$ axis and the BLER ensured by the cooperation on the $\mathrm{Y}$ axis. These results are compared to the ones obtained by simulations and a good match between them can be noticed. Both simulated and theoretical results show that the BLER gain provided by the cooperation protocol depends only on the cooperation probability, if the direct link's BLER is small and the cooperation probability is smaller than one.

If the cooperation probability equals one the BLER gain increases more abruptly with the decrease of the direct link BLER. The slope of this curve tends to the diversity order of the users involved in cooperation, i.e. the number of check blocks +1 , as the direct links BLER decrease. The simulations performed showed that by increasing the relay link's SNR the slope of the BLER vs. BLER curves increases slightly only for high BLERs on the direct link.

The BLER on the direct links and the cooperation probability cannot be completely separated. The link adaptation of the direct UT-BS transmission affects the transmission over the source - relay link, both transmissions taking place in the same broadcast phase. One should also notice that any modification of the direct transmission will affect the overall performance when employing cluster cooperation.

\section{PERformance EVAluation of the RS Coded COOPERATIVE TRANSMISSION}

The parameters of the considered cooperation protocol are summarized in Table I.

Due to the better positioning of the relay node relatively to the BS, an improvement of the SNR of the RN-BS with $4 \mathrm{~dB}$ is considered. Figure 5 and Figure 6 present the BLER and BER performance ensured by the RS-coded cooperation for different cooperation probabilities, when all user terminals have identical UT-RN and UT-BS links. These performances are identical for all UTs in this particular situation.

The results obtained by computer simulations show a significant improvement of the "waterfall" behavior of the combined channel - network code, when the cooperation probability is one. If the cooperation probability decreases, the slope of the BLER vs. SNR curve decreases as well, while if the SNR goes above a given threshold, the slope of the curve becomes similar to that of the channel code. This threshold value and the obtained coding gain decrease as the cooperation probability decreases. These results can be explained by the relation existing between the cooperation probability and the diversity gain provided by the RS-coded cooperation protocol.

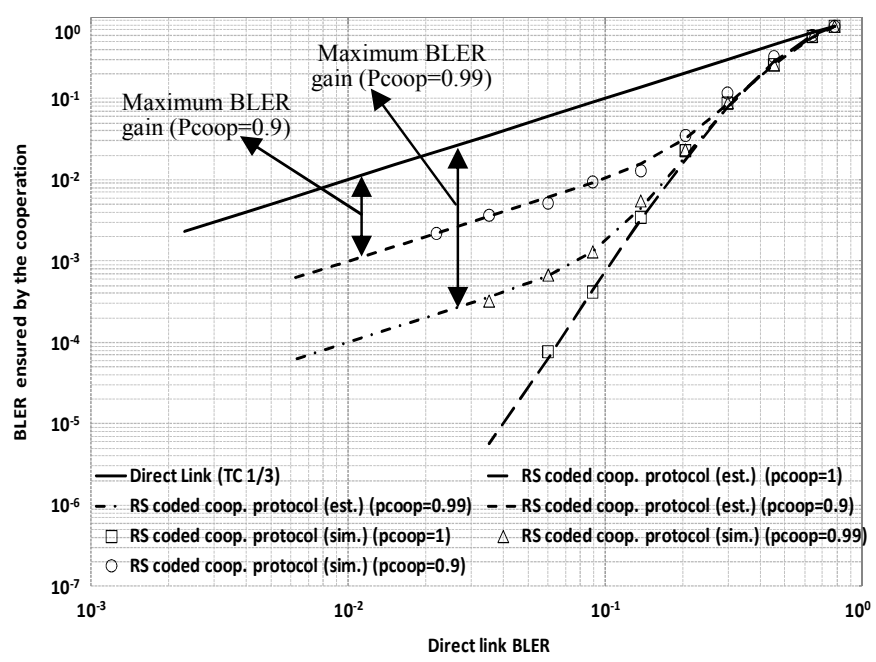

Figure 4. BLER ensured by the cooperation scheme vs. BLER obtained by the direct transmission 
TABLE I The Parameters of the COOPERATION Cluster

\begin{tabular}{|c|c|}
\hline \multicolumn{2}{|l|}{ Direct Transmission (UT-BS, RN-BS) } \\
\hline Channel Code & LTE Turbo Code \\
\hline Coding rate & $1 / 3$ \\
\hline Interleaver length & 1600 \\
\hline Signaling & BPSK \\
\hline Cluster code & RS $(15,11)$ \\
\hline Number of user terminals in cluster & 8 \\
\hline $\begin{array}{l}\text { Number of check blocks transmitted by } \\
\text { the RN }\end{array}$ & 4 \\
\hline
\end{tabular}

If the cooperation probability is one, we have a significant diversity gain provided by the cooperation protocol. This has an important influence over the "waterfall" behavior of the combined channel - network code. On the other hand, if the cooperation probability is less than one, for small direct link BLER values no diversity gain will be provided by the use of the network code. In this latter case the "waterfall" behavior of the combined code is not improved and only a coding gain will be provided by the cooperation protocol. The value of this coding gain depends only on the cooperation probability.

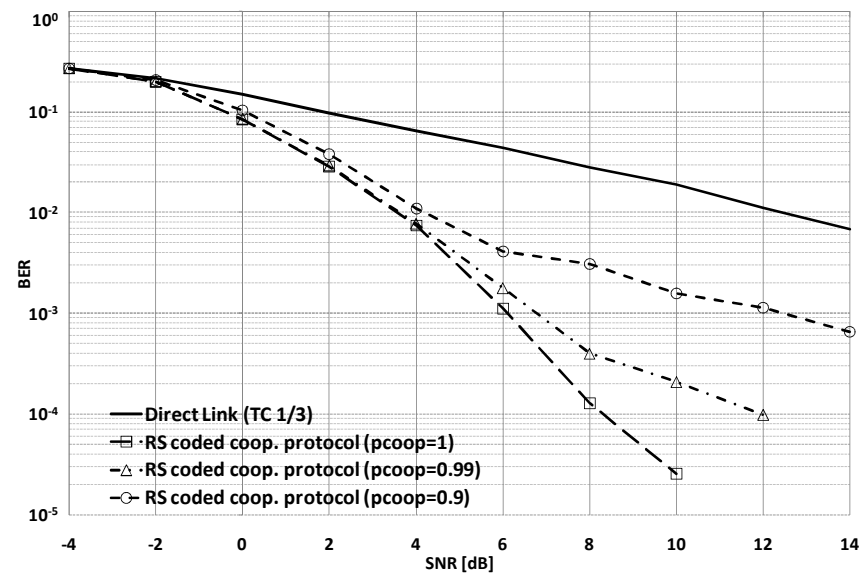

Figure 5. BER performance of the RS-coded cooperation scheme

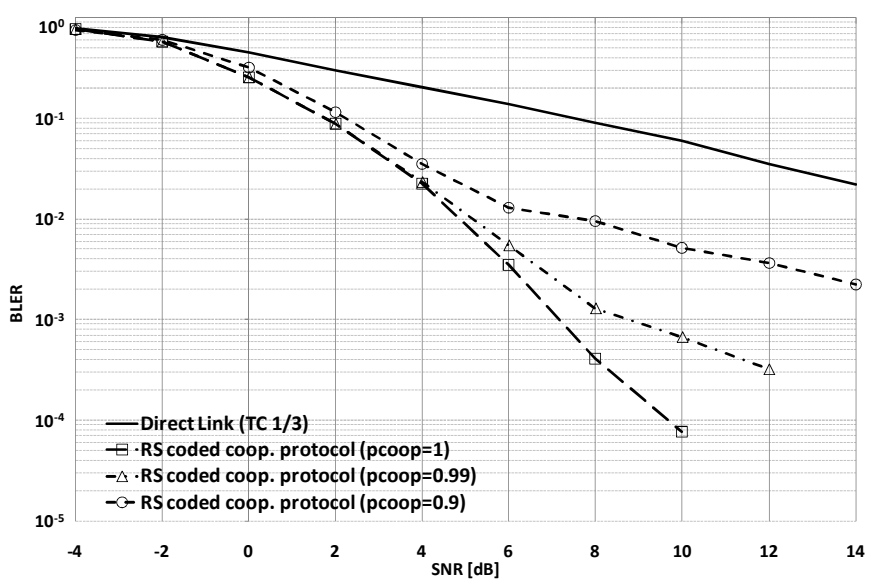

Figure 6. BLER performance of the RS-coded cooperation scheme
Based on the simulation results we conclude that acceptable improvement of the performance in the quasi-static fading scenario requires a cooperation probability greater than 0.9. Significant improvement of the "waterfall" behavior over quasi-static fading channels requires the cooperation probability to be at least around 0.99 . It is important to notice the relative independence of the channel and cluster codes, which allows a simplification of the code design process. Appropriate selection of the channel code and relay assignment need to ensure a large cooperation probability, while the selected cluster code has to provide large diversity and coding gain, in the limits of the resources available.

\section{CONCLUSIONS}

The paper proposes a network coded cooperation protocol for the uplink transmission of a relay enhanced cellular network with non-ideal source-relay links. This cooperation method is a particular one, requiring only one relay per cluster and no connectivity between UTs, which makes it suitable for integration in cellular systems.

The cooperation uses the Reed Solomon codes as network codes, thus avoiding the problems characteristics to graphbased codes. The BLER performance of the proposed protocol is evaluated both theoretically and by computer simulations. The evaluation results demonstrate a significant BLER improvement that can be provided by this approach. This improvement is quantified by the BLER gain parameter, which was also defined and computed. The study is performed considering UT - RN channels affected by errors, whose BLER values are included in the BLER performance analysis.

\section{REFERENCES}

[1] B. Zhao and M. C. Valenti, "Distributed Turbo Coded Diversity for Relay Channel", Electronics Letters, vol. 39, no. 10 pp. 786-787, 15 May 2003.

[2] A. Nosratinia, T. E. Hunter and A. Hedayat, "Cooperative Communication in Wireless Networks", IEEE Communications Magazine, vol. 42, no. 10, pp. 74-80, Oct. 2004.

[3] J. N. Laneman, D. N. C. Tse, and G. W. Wornell, "Cooperative Diversity in Wireless Networks: Efficient Protocols and Outage Behavior", IEEE Trans. Inform. Theory, vol. 50, no. 12, pp. 3062-3080, Dec. 2004.

[4] C. Hausl and P. Dupraz, "Joint Network-Channel Coding for the Multiple-Access Relay Channel”, International Workshop on Wireless Ad Hoc and Sensor Networks, New York, USA, June 2006.

[5] S. Zhang, Y. Zhu, S. C. Liew, and K. Ben Letaief, "Joint Design of Network Coding and Channel Decoding for Wireless Networks", IEEE WCNC 2007, Hong Kong, March 11-15, 2007.

[6] Y. Chen, S. Kishore, and J. (Tiffany) Li, "Wireless Diversity through Network Coding”, IEEE WCNC 2006, Las Vegas, NV, March 2006.

[7] X. Bao and J. (Tiffany) Li, "Matching Code-on-Graph with Network-onGraph: Adaptive NetworkCoding for Wireless Relay Networks", 43rd Allerton Conf. Communication, Control, and Computing, Urbana Champaign, IL, Sept. 2005.

[8] J. Zhang, and T. M. Lok, "Cooperative Protocols for Multiple-Source Multiple-Relay Wireless Networks", International Journal of Sensor Networks, vol. 4, no. 4, pp. 209-219, 2009.

[9] I. S. Reed and G. Solomon, "Polynomial Codes Over Certain Finite Fields," SIAM Journal of Applied Math., vol. 8, pp. 300-304, 1960.

[10] A. G. Fabregas and G. Caire, "Turbo-like Codes are Good for the BlockFading Channel", ISITA 2004 International Symposium on Information Theory and Applications, Parma, Italy, Oct. 10-13, 2004. 